\title{
TRANSFER-MATRIX SIMULATIONS OF LINEAR MAGNETIC SYSTEMS
}

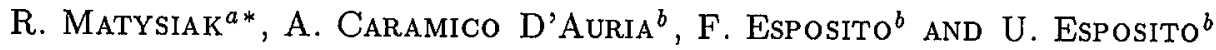 \\ ${ }^{a}$ Computational Physics Division, Institute of Physics, A. Mickiewicz University \\ Umultowska 85, 61-614 Poznań, Poland \\ ${ }^{b}$ Dipartimento di Scienze Fisiche, Università di Napoli \\ Piazzale Tecchio, 80125 Napoli, and INFM Unità di Napoli, Italy
}

\begin{abstract}
The quantum transfer-matrix method was applied to study the finite-temperature static properties of the spin $S=1$ antiferromagnetic Heisenberg chains in a wide range of the single-ion anisotropy and temperatures. The high-resolution quantum transfer-matrix simulation data are obtained for the zero-field susceptibility, specific heat as well as for the field-dependent magnetization. The microscopic parameters of a number of real quasi-one-dimensional compounds are found from fitting procedures, some theoretical approaches are numerically verified and an extension of the technique to a non-uniform bond alternating molecular magnets is also put forward.
\end{abstract}

PACS numbers: $75.10 . \mathrm{Jm}, 75.40 . \mathrm{Cx}, 75.40 . \mathrm{Mg}$

\section{Introduction}

Antiferromagnetic spin chains have attracted a lot of theoretical and experimental interest due to their intrinsic quantum properties. The ground state of integer spin chains was predicted disordered with a gap in the excitation spectrum and the spin-correlation function decaying exponentially. The existence of the gap has been well established by various numerical techniques and field-theoretical arguments. The representative physical realizations of the one-dimensional Heisenberg antiferromagnets (1D HAF) $S=1$ with varying anisotropies are the compounds: NENP [1], YBANO [2], TMNIN [3] and $\mathrm{AgVP}_{2} \mathrm{~S}_{6}$ [4].

In recent years a number of new quasi-one-dimensional molecular-based magnets has been synthesized. These compounds are characterized not only by uniform antiferromagnetic but also non-uniform alternating-bond interaction [5-9].

The aim of this report is to present numerical simulations based on the quantum transfer-matrix (QTM) technique applicable to 1D HAF to verify some theoretical results and to compare the simulations with the corresponding experimental data.

\footnotetext{
*e-mail: matysiak@pearl.amu.edu.pl
} 
We consider the following bond-alternating $S=1$ Hamiltonian

$$
\mathcal{H}=J_{1} \sum_{i=1}^{N / 2} S_{2 i-1} \cdot S_{2 i}+J_{2} \sum_{i=1}^{N / 2} S_{2 i} \cdot S_{2 i+1}+D \sum_{i=1}^{N}\left(S_{i}^{z}\right)^{2}-g_{\nu} \mu B \sum_{i=1}^{N} S_{i}^{\nu},
$$

where $J_{1}, J_{2}$ denote the nearest-neighbour interaction constants. We assign the positive values to $J_{i}(i=1,2)$ for the antiferromagnetic coupling. $D$ stands for the single-site anisotropy parameter, $B$ is the external magnetic field which can be applied along the chain $(\nu=z)$ or in the perpendicular direction $(\nu=x), g_{\nu}$ is the corresponding gyromagnetic ratio and $N$ is the size of the system.

\section{The quantum transfer-matrix technique}

We calculate both the canonical partition function defined as $\mathcal{Z}=\operatorname{Tr} \mathrm{e}^{-\beta \mathcal{H}}$, and the thermodynamical mean values of a quantity described by the self-adjoint operator $\mathcal{A}$ which is given by $\langle\mathcal{A}\rangle=\frac{1}{\mathcal{Z}} \operatorname{Tr} \mathcal{A} \mathrm{e}^{-\beta \mathcal{H}}$. We introduce the operator $\mathcal{R}(\beta)=\prod_{i=1}^{N} \mathrm{e}^{-\beta \mathcal{H}_{i, i+1}}$, where $\mathcal{H}_{i, i+1}$ represents an interaction Hamiltonian for the spin pair $\left(S_{i}, S_{i+1}\right)$. Using the general Suzuki-Trotter formula, the partition function $\mathcal{Z}$ can be expressed in terms of $\mathcal{R}$

$$
\mathcal{Z}=\lim _{m \rightarrow \infty} \mathcal{Z}_{m}=\lim _{m \rightarrow \infty} \operatorname{Tr}[\mathcal{R}(\beta / m)]^{m},
$$

where $m$ is the natural (Trotter) number and $\mathcal{Z}_{m}$ is the $m$-th approximant to $\mathcal{Z}$. We note that $\mathcal{Z}_{m}$ can be calculated numerically without any restrictions on the value of $N$ by the quantum transfer-matrix method [10,11], changing the transfer direction. The thermodynamical functions can be found by the derivatives of $\mathcal{Z}$ or, more efficiently, from the corresponding mean values (e.g. the internal energy or the field-dependent magnetization).

\section{Results}

In the isotropic limit, we calculated both the susceptibility and specific heat as well as the field-dependent magnetization. Extensive large-scale simulations in the interval of parameters appropriate for NENP and other quasi-one-dimensional chains were also performed [10]. The best-fit parameters found for NENP, $\mathrm{CsNiCl}_{3}$, and YBANO are the following: $J / k_{\mathrm{B}}=48 \mathrm{~K}, D / k_{\mathrm{B}}=7.8 \mathrm{~K}, g_{\perp}=2.25$, $g_{\|}=2.20 ; J / k_{\mathrm{B}}=27 \mathrm{~K}, D / k_{\mathrm{B}}=1.35 \mathrm{~K}, g=2.23 ; J / k_{\mathrm{B}}=275 \mathrm{~K}, D / k_{\mathrm{B}}=60 \mathrm{~K}$, $g=2.33$, respectively. The simulations also imply some theoretical consequences as far as the boson and fermion approximations and strong anisotropy calculations are concerned [10].

We have also analyzed a number of uniform and bond-alternating molecularbased chains: (a) the compound trans-[Ni(333-tet $\left.)\left(\mu-\mathrm{N}_{3}\right)\right]_{n}\left(\mathrm{ClO}_{4}\right)_{n}$ - denoted as 1 by [5] and abbreviated here as $A 1 ;$ (b) $\left[\mathrm{FeON}_{8} \mathrm{C}_{26} \mathrm{H}_{14}\right]_{n}$ - considered by [6] and abbreviated here as $A 2$; (c) the compounds 1 and $2-$ considered by [7] and abbreviated here as $A 3$ and $A 4$, respectively; (d) $\left[\mathrm{Ni}\left(\mathrm{N}_{3}\right)_{2} \text { (tmeda) }\right]_{n}$ - considered by [8] and abbreviated here as $A 5$; (e) $\mathrm{Ni}_{2}$ (EDTA) $\cdot 6 \mathrm{H}_{2} \mathrm{O}$ considered by [9] and abbreviated here as $A 6$. It is worth noting that for $A 6$, both the experimental susceptibility and the magnetic specific heat data are reported [9]. The latter are difficult to extract and are subject to experimental uncertainties so that they are 


\section{TABLE}

Estimates of the best-fit exchange couplings $J_{1}$ and $J_{2}$ and the $g$ factor for the compounds listed in the first column.

\begin{tabular}{c|c|c|c}
\hline \hline$A n$ & $J_{1} / k_{\mathrm{B}}[\mathrm{K}]$ & $J_{2} / k_{\mathrm{B}}[\mathrm{K}]$ & $g$ \\
\hline$A 1$ & $99.5 \pm 2.0$ & $63.5 \pm 2.0$ & $2.38 \pm 0.02$ \\
$A 2$ & $408.0 \pm 0.0$ & $90.0 \pm 15$ & $2.33 \pm 0.02$ \\
$A 3$ & $225 \pm 5.0$ & $-25.0 \pm 5.0$ & $2.45 \pm 0.03$ \\
$A 4$ & $40 \pm 1.0$ & $25.0 \pm 5.0$ & $2.39 \pm 0.02$ \\
$A 5$ & $43.0 \pm 1.0$ & $13 \pm 0.5$ & $2.35 \pm 0.02$ \\
$A 6$ & $10.6 \pm 1.0$ & $3.3 \pm 0.5$ & $2.20 \pm 0.02$
\end{tabular}

usually not a vailable. Our accurate QTM simulations for the compounds $A 1-A 6$ and the subsequent fitting analyses to the experimental susceptibility data lead to the values for the best-fit parameters listed in Table.

Our results for $A 6$ are presented in Figs. 1 and 2 as the temperature dependence of the molar susceptibility in [memu/mole] and the specific heat in dimensionless units, respectively. The measured values are plotted with the diamonds and the best QTM fit is drawn with the dotted line. They reveal a pronounced anisotropy $D=5 \pm 1 \mathrm{~K}$.

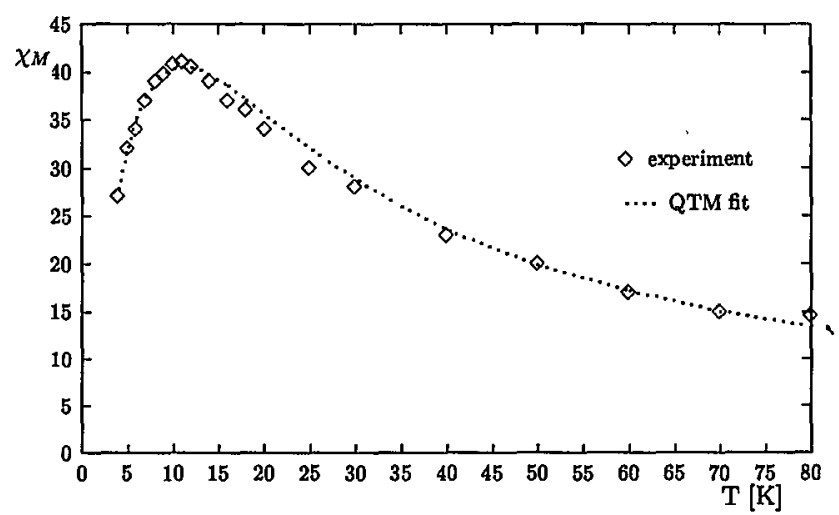

Fig. 1. The molar susceptibility $\chi_{M}$ in [memu/mole] versus temperature for $A 6$. The measured values are plotted with the diamonds and the best QTM fit (with $J_{1}=$ $-10.6 \pm 1.0 \mathrm{~K}, J_{2}=-3.3 \pm 0.5 \mathrm{~K}, g=2.20 \pm 0.02, D=5 \pm 1.0 \mathrm{~K}$ ) is drawn with the dotted line.

As far as the susceptibility is concerned, for all the alternating chains the agreement between experiment and theory is within $3 \div 4 \%$. We also demonstrate in Fig. 2 that our estimates of the specific heat are consistent with experiment for the same couplings. At low temperatures, the deviations to some extent might be attributed to the uncertainties of our numerical data (lower than $5 \%$ above $T=5 \mathrm{~K}$ ), whereas for higher temperatures this explanation should be ruled out. 


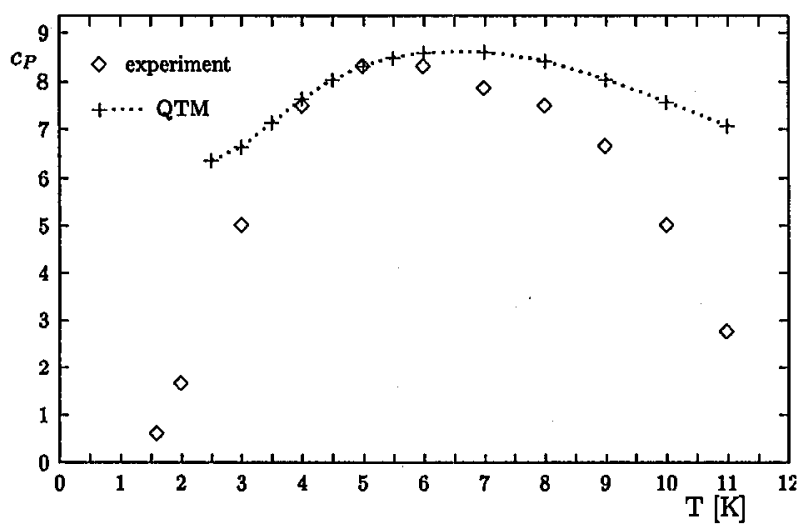

Fig. 2. The specific heat of $A 6$. The measured values are plotted with the diamonds and the best QTM fit (with $J_{1}=-10.6 \pm 1.0 \mathrm{~K}, J_{2}=-3.3 \pm 0.5 \mathrm{~K}, g=2.20 \pm 0.02, D=$ $5 \pm 1.0 \mathrm{~K})$ is drawn with the dotted line.

We would like to thank Professors Dante Gatteschi and Grzegorz Kamieniarz for helpful discussion. This work was supported in part by the Committee for Scientific Research (Poland) under grant No. 2 P03B 058 14. Numerical calculations were mainly carried out on the platforms of the Supercomputing and Networking Center in Poznań.

\section{References}

[1] J.P. Renard, M. Verdagner, L.P. Regnault, W.A.C. Erkelens, J. Rossat-Mignad, J. Ribas, W.G. Stirling, C. Vettier, J. Appl. Phys. 63, 3538 (1988).

[2] T. Shimizu, D.E. MacLaughlin, P.C. Hammel, J.D. Thompson, S.-W. Cheong, Phys. Rev. B 52, R9845 (1995).

[3] V. Gadet, M. Verdagner, V. Briois, A. Gleizes, J.P. Renard, P. Beauvillain, C. Chappert, T. Goto, K. Le Dang, P. Veillet, Phys. Rev. B 44, 705 (1991).

[4] T. Asano, Y. Ajiro, M. Mutka, H. Yamazaki, N. Hosoito, T. Shinjo, H. Kikuchi, Solid State Commun. 90, 125 (1994).

[5] A. Escuer, R. Vicente, J. Ribas, M. Salah El Fallah, X. Solans, M. Font-Baria, Inorg. Chem. 33, 1842 (1994).

[6] W. Hiller, J. Strähle, A. Datz, M. Hanack, W.E. Hatfield, L.W. ter Haar, Ph. Gütlich, J. Am. Chem. Soc. 106, 329 (1984).

[7] J. Ribas, M. Monfort, B.K. Ghosh, X. Solans, M. Font-Bardia, J. Chem. Soc., Chem. Commun., 2375 (1995).

[8] J. Ribas, M. Monfort, B.K. Ghosh, X. Solans, Angew. Chem. Int. Ed. Engl. 33, 2087 (1994).

[9] J.J. Borras-Almenar, E. Coronado, J. Curely, R. Georges, Inorg. Chem. 34, 2699 (1995).

[10] G. Kamieniarz, R. Matysiak, A. Caramico D'Auria, F. Esposito, U. Esposito, Phys. Rev. B 56, 645 (1997).

[11] F. Esposito, G. Kamieniarz, Phys. Rev. B 57, 7431 (1998). 